\title{
Control Parameter Setting of IEEE 802.11e for Proportional Loss Rate Differentiation
}

\author{
Seung-Jun Lee, Chunsoo Ahn, and Jitae Shin \\ School of Information and Communication Engineering, Sungkyunkwan University, \\ Suwon, 440-746, Korea \\ $\{1$ j6467, navy12, jtshin\}@ece.skku.ac.kr
}

\begin{abstract}
The IEEE 802.11 DCF mechanism does not present performance differentiation, because Best-effort-Service is used for the degree of importance of packets. The Enhanced Distributed Coordination Function (EDCF) mechanism of IEEE 802.11e supports QoS. According to the degree of importance of packets, packets are assigned priority and control parameters are assigned difference values. Through differentiation of theses parameters, differentiated services can be provided to various priority packets (classes) in terms of throughput, packet loss rate, and delay. In this paper, parameters of the IEEE 802.11e EDCF mechanism for Proportional Loss Rate Differentiation Service(PLDS) between adjacent priority classes are investigated through mathematical analysis and network simulation.
\end{abstract}

\section{Introduction}

The DCF mechanism only provides a best-effort service, even though the importance of packets or type of packets (real-time or non real-time) exists [1.IEEE 802.11e Enhanced DCF (EDCF) supports QoS. The MAC method of the EDCF mechanism is similar to the DCF, with the exception that EDCF applies a different value to control parameters, according to the packet type, to support QoS 3. The Markov chain model is used frequently for mathematical analysis of the IEEE 802.11 mechanism [2]. However, Markov chain analysis in [2] had two problems, ignoring the cases of dropping packets and the frozen slot time. Ref. [4] uses a modified Markov chain model for mathematical analysis of the IEEE 802.11e EDCF mechanism. In the case of [4,this includes two cases that are not considered in [2]. In this paper, based on [4] and [5], we will find out which control parameters such as the $\mathrm{CW}_{\min } / \mathrm{CW}_{\max }$, retry limit, AIFS, and so on., in IEEE 802.11e EDCF mechanism have the most effect on loss rate. In addition, in order to obtain a proportional and differentiated performance between adjacent priority classes, a method of setting the most dominant parameter in QoS, is proposed.

\section{Mathematical Model of Packet Loss Rate}

In order to support QoS, IEEE 802.11e EDCF classifies the packets and the packet map into four access categories (ACs), according to priority. AC 
denotes $\mathrm{AC}[i](i=0,1,2,3)$ for distinguishing priority. A smaller value of $i$ represents higher priority. Each $\mathrm{AC}[i]$ is assigned different values of parameters $\left(\mathrm{CW}_{\min }[i] / \mathrm{CW}_{\max }[i], \mathrm{AIFS}[i]\right.$, etc $)$. A default value of IEEE 802.11e EDCF parameters is defined in $[3$.

The backoff stage increases by one, in the case where a station does not transmit a packet. If collision occurs continuously, the backoff stage continues to increase. As presented in Fig. 1 of ref. [5], the backoff stage increases until the maximum retry limit, i.e., $L$. At the maximum retry limit, if the packet collision occurs with the backoff stage, and it is the same as the value of maximum retry limit, the packet will be dropped. The packet-loss-rate is defined by the following equation.

Let $P_{i, \text { loss }}(i=0,, \mathrm{~N}-1)$ denote the packet-loss probability for the priority $i$ class.

$$
P_{i, \mathrm{loss}}=p_{i}^{L_{i}+1}
$$

As $p_{i}$ of Eq. (1) is the probability that a transmitted packet collides, refer to Eq. (9) of ref. [5].

\section{Analysis of Proportional Differentiation Service in Loss Rate}

In Proportional Loss Rate Differentiation Service(PLDS), the goal is to have the packet loss rate ratio between the adjacent $i$-th priority class and $i+1$-th priority class, at a certain value $\left(K_{L}\right)$ such as Eq. (2).

$$
\frac{P_{i+1, \mathrm{loss}}}{P_{i, \mathrm{loss}}}=\frac{p_{i+1}^{L_{i+1}+1}}{p_{i}^{L_{i}+1}} \triangleq K_{L}
$$

The desired loss rate ratio is assigned a $K_{L}$. When taking the logarithm to Eq. (2),

$$
\log K_{L}=\left(L_{i+1}+1\right) \log p_{i+1}-\left(L_{i}+1\right) \log p_{i}
$$

Arrange Eq. (3) in terms of $L_{i+1}$. Then $\log p_{i}$ and $\log p_{i+1}$ are considered as nearly same value for our approximation. Therefore,

$$
L_{i+1} \cong L_{i}+\frac{\log K_{L}}{\log p_{i+1}}=L_{i}+\frac{\log K_{L}}{a(n) \log n}
$$

Where $n$ and $a(n)$ are the number of nodes and a related constant value to compensate the relationship between $\log p_{i+1}$ and $\log n$, respectively. From Eq. (4), guidance of PLDS between adjacent classes can be derived. When the value of $L_{i}$ is provided, the $K_{L}$ for the desired ratio between adjacent priority classes is assigned, and the collision probability $p_{i+1}$ calculated according to the station number $(n)$ is obtained, $L_{i+1}$ can be found out, and should be set for PLDS. 


\section{Numerical and Simulation Results}

In this section, the validation of PLDS through numerical and simulation results is provided. For basic parameter setting, the IEEE 802.11 FHSS system parameters of ref. 2] are referred to.

In both numerical and simulation by the NS-2 simulator, priority classes are divided into classes 0,1 , and 2 (class 0 represents the highest priority). The number of each priority class station is assumed to be identical. The numerical and simulation results are performed at the number of each priority class station, from 4 to 15 . That is, the number of total stations (or nodes) varies from a minimum value of 12 to a maximum value of 45 nodes.

In order to present validation of PLDS, except for retry limit $\left(L_{i}\right)$, which most influencing parameter for PLDS, it is assumed that other control parameters remains as the same cross classes. The $\mathrm{CW}_{\min }$ of all classes is 16 , maximum backoff stage of all class is 2 , and initial value of $L_{0}$, i.e., maximum retry limit of the highest priority class 0 , is fixed at 4 . When the node number is counted and the ratio $\left(K_{L}=2\right)$ for PLDS is provided, then $L_{1}$, i.e., 3 , and $L_{2}$, i.e., 2 , can be calculated through Eq. (2). However the calculated value through Eq. (2) is not the exact difference with the number 1 . The result is actually a decimal smaller than the number 1 . However, the value of the maximum retry limit should have an exact positive number. Therefore, the difference of the retry limit between adjacent priority classes is approximately applied as 1. Fig. 1(a) presents numerical results for the loss rate of classes and Fig. 1(b) presents the simulation results for loss rate of classes. When Fig. 1(a) is compared with Fig. 1(b)] little difference exists. This is due to NS-2 random execution. In addition, Fig. 1(c) (regarding analysis) and Fig. 1(d)(regarding simulation) confirms whether the desired ratio is obtained through PLDS.

When the number of the station (or node) is minimal, i.e., the nodes for each class consist of approximately $2 \sim 6$ numbers, PLDS deviates considerably from the desired ratio. This affects the absolute value of $\log p$.

\section{Conclusion}

In this paper, mathematical analysis of the loss rate for the proposed PLDS is derived, in order to provide proportional and differentiated services among different priority classes in the IEEE $802.11 \mathrm{e}$ mechanism. In addition, validation of PLDS is proven through NS-2 simulation results.

As a result, the desired loss-rate according to the type of packets is provided in the case where data is transmitted. The maximum retry limits are very sensitive to packet loss, and are applied differently to each packet, depending on the type of transmitted packets. Therefore, users can directly control the condition of a network. If the packet should not be lost, a larger maximum retry limit is applied to recover packet loss. In addition, if the packet is less affected by dropping, the packet takes maximum retry limit that is smaller than that of sensitive packet. Through this technique, the complexity of a network can be reduced. 


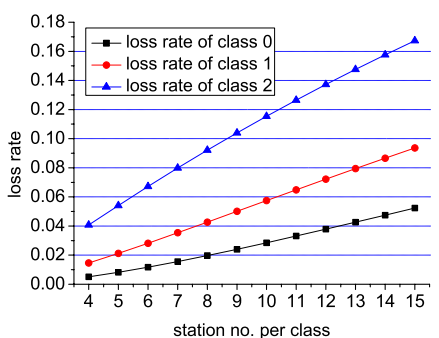

(a) Analysis: $K_{L}=2\left(L_{0}=4, L_{1}=\right.$ $3, L_{2}=2$ )

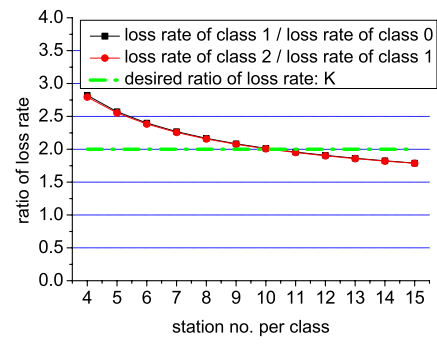

(c) Analysis: loss rate ratio

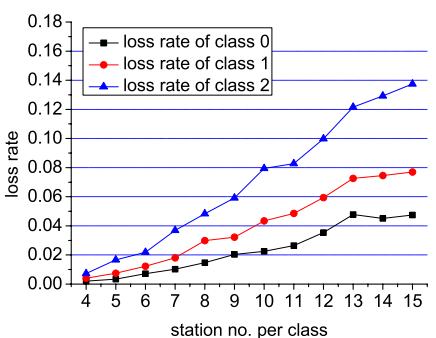

(b) Simulation: $K_{L}=2\left(L_{0}=4\right.$, $L_{1}=3, L_{2}=2$ )

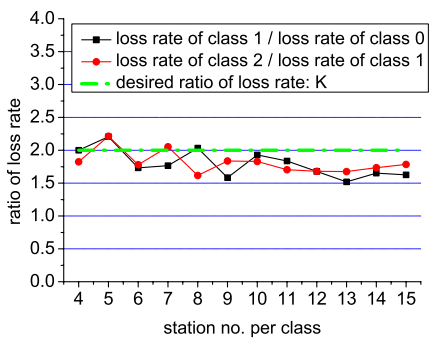

(d) Simulation: loss rate ratio

Fig. 1. Analysis and simulation results

\section{Acknowledgements}

This research was supported by the Ministry of Information and Communication (MIC), Korea, under the Information Technology Research Center (ITRC) support program supervised by the Institute of Information Technology Assessment (IITA) (IITA-2005-(C1090-0502-0027)).

\section{References}

1. IEEE Standard for Wireless LAN Medium Access Control(MAC) and Physical Layer(PHY) Specifications, P802.11, Nov. 1997.

2. G. Bianchi, "Performance Analysis of the IEEE 802.11 Distributed Coordination Function," IEEE Journal on Selected Areas in Communications, vol. 18, no. 3, pp. 535-547, Mar. 2000.

3. IEEE 802.11 WG, Draft Supplement to Part 11: Wireless Medium Access Control (MAC) and physical layer (PHY) specifications: Medium Access Control (MAC) Enhancements for Quality of Service (QoS), IEEE 802.11e/D2.0, Nov. 2001.

4. Yang Xiao, "Performance Analysis of IEEE 802.11e EDCF under Saturation Condition,"IEEE Communications Society, 2004.

5. Seung-Jun Lee, Chunsoo Ahn, and Jitae Shin, "Control Parameter Setting of IEEE 802.11e for Proportional Throughput," ICOIN 2006. 